\title{
Bachelor groups form due to individual choices or environmental disrupters in
}

\section{African striped mice}

3 Sthandiwe Nomthandazo Kanyile ${ }^{1,2}$, Neville Pillay ${ }^{1}$, Carsten Schradin ${ }^{1,3}$,

$4{ }^{1}$ School of Animal, Plant and Environmental Sciences, University of the Witwatersrand, South Africa.

$5{ }^{2}$ Department of Evolutionary Ecology, Institute of organismic and Molecular Evolution, Johannes

6 Gutenberg University, Mainz, Germany

7 3 Université de Strasbourg, CNRS, IPHC UMR 7178, F-67000 Strasbourg, France

8 Corresponding author :Sthandiwe Nomthandazo Kanyile, email : 2012153603@ufs4life.ac.za

9

10

\section{PUBLISHED AS}

Kanyile, S. N., Pillay, N. \& Schradin, C. 2021. Bachelor groups form due to individual choices or environmental disrupters in African striped mice. Animal Behaviour, 182, 135-143.

\begin{abstract}
In several mammal species, bachelor groups occur as a regular life history stage between dispersal and becoming the breeding male of a multi-female group. However, it is rarely investigated how such groups come into existence and how males that choose this strategy differ in life history traits from other males. Males of the socially flexible African striped mouse Rhabdomys pumilio have been historically reported to adopt one of three alternative tactics, i.e., small group-living philopatric males, intermediate solitary living roaming males, or large group-living territorial males. Here, we describe for the first time, bachelors as a fourth male tactic. Using long-term data from 13 generations of striped mice we show that there are in fact two types of bachelor groups: first, kin bachelor groups formed when all females disappeared, probably due to predation; and second non-kin bachelor groups formed by unrelated males that originate from different groups. Whereas kin bachelor groups occurred more frequently during the non-breeding season, non-kin bachelor groups were most common in the breeding season and had a shorter tenure, since most of these males soon switched to the solitary roaming tactic or the territorial breeder tactic. Importantly, we found that non-kin bachelors were heavier and older than kin bachelors and that they did not differ from solitary roaming males in body mass and age. In summary, we identified two different forms of bachelor groups, kin bachelors whose female group members disappeared and larger non-kin bachelor groups showing traits of an alternative reproductive tactic. Both types of bachelor groups differed in multiple traits, demonstrating that it is important to follow the life histories of individuals to understand the mechanisms leading to alternative tactics.
\end{abstract}

Keywords: alternative reproductive tactics; male-male groups; reproductive success; social flexibility; strategy; thermoregulation 
The social organisation of a species describes its composition of social units and is an important part of its social system (Kappeler 2019; Kappeler \& Schaik 2002). Species vary in their social organisation from solitary to pair- and- group-living (Shultz, Opie \& Atkinson 2011). Variation also occurs within species, especially when both males and females follow alternative social tactics (Lott 1991; Schradin 2013). Social organisation can be sex-specific; for example, herd living females and mainly solitary males in African elephants (Loxodonta africana), (O'Connell-Rodwell et al. 2011) as well as some artiodactyls (Jaeggi et al. 2020). Groups of bachelor males are another example of a sex specific form of social organisation and have been described mainly in medium sized to large mammals.

While there is no generally agreed upon definition of bachelor males, we regard all groups that consist of males only as bachelor groups, independent of the (often unknown) reproductive consequences of being a bachelor male (Roberts 2017), but excluding male groups that meet primarily for mating and breeding, such as leks (Westcott 1994) and colonies (Robisson, Aubin \& Bremond 1993). In many species, larger males monopolise access to groups of breeding females. Since the sex ratio in mammals at birth is close to $1: 1$, the remaining males must live either solitarily or form bachelor groups that consist of males with low competitive abilities as exemplified in species such as Hanuman langurs (Semnopithecus entellus; Koenig \& Borries 2001), geladas (Theropithecus gelada; le Roux \& Bergman 2012), white rhinoceros (Ceratotberium simum; Gartlan 1974), and many antelopes (Jarman 1974; Wirtz 1982). In these species, there is often only one big competitive breeding male per female group, and the remaining males are evicted by the dominant male from the groups at adolescence, being forced into bachelor groups for predator avoidance (Waterman 1997). Bachelor groups seem to occur mainly in mammals, although some have been reported in other taxa. In eider ducks (Somatetria mollissima) males leave the females after mating and form bachelor groups, while females incubate alone and raise chicks in creches (Burton \& Burton 1969). Reports for small mammals below $1 \mathrm{~kg}$, which represent the majority of all mammal species, are also rare. The smallest mammal known to have bachelor groups are Cape ground squirrels with a body mass of about $1 \mathrm{~kg}$ (Waterman 1997; Wirtz 1982).

Bachelor males are often overlooked in behavioural studies, even where their existence has been observed. For example, in patas monkeys (Erythrocebus patas), bachelor groups have been known for decades (Gartlan 1974) but have received rather little attention thereafter (Ohsawa 2003). Subsequently, the mechanisms leading to the formation of bachelor groups are rarely studied, since in most species it is simply assumed to be an automatically occurring life history stage of waiting males. However, becoming a bachelor is not obligate in all species where bachelor groups occur, and studying how bachelors differ from other males might help us to understand why males choose the bachelor tactic over an alternative tactic. Two possibilities may theoretically give rise to bachelor groups. 1) Environmental disrupters (Schradin 2013) can lead to the formation of bachelor groups when all females of a group die and leave only males behind. While the existence of such bachelor groups would require no further evolutionary explanation as males become bachelors due to stochastic extrinsic factors and not due to individual choice; the observed behaviour of males remaining in a group after all females disappear would still be considered a tactic, albeit, arising from a different mechanism. 2) Alternatively, males could leave mixed sex groups to form bachelor groups. In such instances, evolutionary explanations would be required to give insights into why males choose to leave their groups, why they would prefer to form a bachelor group instead of being solitary and how this ultimately influences their fitness. It has previously been shown that distinguishing whether females chose a solitary breeding tactic or became solitary due to the death of all other group females (environmental disrupter) has important consequences for understanding differences in body mass and stress hormone levels (Hill, Pillay \& Schradin 2015a, b).

Here we report, to our knowledge for the first time, the occurrence of bachelor males in a mammal with a body mass below $100 \mathrm{~g}$, namely the socially flexible striped mouse Rhabdomys pumilio from the arid Succulent Karoo (Schradin et al. 2012). In this species, males can follow alternative social and 
reproductive tactics (Schradin et al. 2009a). Males are born in the spring breeding season and remain philopatric within their natal group throughout the dry non-breeding season in summer. In the following winter and the spring breeding season, some males remain philopatric, but most males leave their natal group, either becoming solitary living roamers or the breeders of groups of communally breeding females (Schradin et al 2009). Philopatric males are the smallest males with very low reproductive success, breeders the largest males with highest reproductive success, and roamers lie in-between (Schradin \& Lindholm 2011). Switching to a tactic of higher reproductive success is associated with having reached a larger body mass and thus higher competitive ability (Schradin \& Lindholm 2011). Group-living is associated with thermoregulatory benefits of sharing a nest (Scantlebury et al. 2006), but striped mice are solitary foragers (Schradin \& Pillay 2004).

The aim of our study was to determine the origin of bachelor groups in African striped mice, and whether bachelor males have traits indicating they represent a waiting tactic (without reproduction) or whether they could represent an alternative reproductive tactic (ART). We expected to identify two types of bachelor males: 1. kin bachelor groups due to environmental disrupters (Schradin 2013), i.e., cases where all female group members had disappeared, leaving the small philopatric males and the breeding male. 2. non-kin bachelor groups consisting of unrelated males originating from different groups. If non-kin bachelor males represent an ART, we hypothesised that they would be scrotal (sexually mature) and occur mainly during the breeding season. We predicted that males of non-kin bachelor groups (but not of bachelor groups arising from environmental disrupters) would be heavier (more competitive) than philopatric males but lighter than breeding males and would be similar in mass to roaming males. We further predicted non-kin bachelors to be more common when population density is high and the adult sex ratio is male biased, since both would increase competition between males, potentially causing males to leave their natal group and form bachelor groups. Finally, we expected bachelor males to switch to the roaming or breeding tactic when their body mass increased, an indicator of increased competitiveness. We predicted that switching from the bachelor tactic should happen first in former breeding males of kin groups (which are already heavy when the bachelor group forms), then non-kin bachelors (predicted to be of intermediate body mass when becoming a bachelor), and finally in philopatric kin males.

\section{MATERIAL AND METHODS}

Study site and period

The study was conducted at the Succulent Karoo Research Station (SKRS) at the Goegap Nature Reserve in the Northern Cape Province, South Africa (S29 41.712 E18 01.5, altitude $912 \mathrm{~m}$ ). This study site receives an average annual rainfall of approximately $160 \mathrm{~mm}$, predominantly in the winter months, where night temperatures often fall below $0^{\circ} \mathrm{C}$, whereas summers are typically hot and dry with temperatures reaching approximately $40^{\circ} \mathrm{C}$ (Schradin and Pillay 2005a). The field site is characterized by sandy areas and patchy bush/shrub distribution (Schradin and Pillay 2004). Data were collected monthly from January 2006 until December 2019, with the exception of April-June 2006.

\section{Trapping, marking, radio-tracking and observation of striped mice}

Individuals were live-trapped using Sherman-like metal traps (26x9x9 cm; Schradin and Pillay 2004). Traps were baited using a mixture of bran flakes, raisins, sea salt and sunflower oil. The traps were placed next to bushes containing the nesting sites of groups or roaming individuals. Trapping was done in the early mornings and in the late afternoons but not during the hottest times of the day, when striped mice are mostly inactive (Schradin et al. 2007). Body mass measurements were taken during both the morning and afternoon trapping sessions, but for this study, only the morning measurements were used for analysis (mice are heavier in the afternoon due to foraging). Traps were checked every 30-45 minutes and trapped mice were weighed (to within $1 \mathrm{~g}$ until August 2010, and to 0.1g thereafter) using an electronic scale. Males were categorized as either scrotal (testes descended) or non-scrotal (testes inside abdomen; Schradin and Pillay 2005b). Each mouse was marked with two ear tags 
137 (National Band and Tag, Newport, KY, USA) and with a unique colour combination of hair dye (Inecto 138 Rapid, Pinetown, South Africa) for identification during behavioural observations. This dye is not 139 known to have any negative effects on the behaviour of striped mice or increase risk of predation 140 (Schradin 2006). Over the years, we monitored between 11 and 16 groups (mean \pm standard deviation; 141 13 \pm 1.68 ). By marking most mice as juveniles, it was possible to record the groups from which most bachelor males originated. Old and unmarked males were assumed to have originated from outside our study site (immigrants); they were ear tagged and marked when they joined the study population.

In each group, at least one breeding female was fitted with a radio-collar throughout the year. In addition, during the breeding season, all breeding males, solitary roaming males, and males whose group association was unknown carried radio-collars (Schradin and Pillay 2005b). Individuals were tracked twice a day (alternating morning/afternoon and at night), five times a week, using an AOR 8000 wide range receiver and a Telonics RA-14K antenna. By tracking individuals to their nesting sites at night, it was possible to record group composition, to identity the males in each group, and to locate nesting sites for behavioural observations of mice not carrying radio-transmitters.

Striped mice typically bask in the sun when they emerge from their nests at sunrise and when they return from foraging at sunset. During this time, it is possible to identify group members due to their individual markings with hair dye, and thus to record group composition. Groups and individuals were directly observed in the mornings (sunrise) and afternoons (sunset) in front of their nests. Each group or individual was observed for two to three consecutive days (both mornings and afternoons), and at least once every two weeks. Observations lasted for 30 to 45 minutes.

\section{Determination of male tactics}

158

159

160

161

162

163

164

165

166

167

168

169

170

171

172

173

174

175

176

177

178

179

180

181

182

183

184

185

A combination of trapping, radio-tracking and direct observations was used to determine the tactics followed by individual males. If males had previously been trapped at a group as juveniles (body mass $<25 \mathrm{~g}$ ) and still trapped as adults in the same group, they were considered philopatric to this group (Schradin and Yuen 2011; Schoepf and Schradin 2012a). Breeding males were identified as the heaviest scrotal male in its non-natal group; there was never more than one non-natal male per group. Males not sharing a nest with any other mice were considered as solitary roamers (Schradin et al. 2012). Two or more males sharing a nest with each other, without any female group member, were identified as bachelor males. Bachelor males were assigned to one of three categories according to relatedness and how the bachelor groups were formed: 1) Kin bachelors: all female members of a group disappeared, leaving behind males, which were related to each other (originate from the same group); 2) Related males of one group which had left their natal group to form a bachelor group. Since this was only observed in one instance where two related males left their natal group to form a bachelor group, these two males were included into the first category for further analysis; 3) Unrelated bachelors: groups consisting of unrelated males that originated from different groups. Thus, we recognised a total of five different male tactics: philopatrics, roamers, breeders, and the two categories of bachelors (kin and non-kin bachelors). Tenure was determined for each bachelor male by counting the number of months that each male continuously shared the same nest with other bachelor males. To determine the body mass at tactic switches, we used the mean body mass of five morning measures before switching to the bachelor tactic or after switching from being a bachelor to next tactic, but within a maximum of 14 days of the tactic switch. The total change in body mass while males were bachelors was measured as the total difference in body mass (g) from the period when males switched to the bachelor tactic and to the period when males changed from bachelor to a different tactic: $\mathrm{g} /$ period).

Determination of population density, breeding intensity, sex ratio and ratio of bachelor males

Population densities were calculated as number of adult striped mice per hectare. We determined population density monthly by dividing the entire number of adult individuals $(>30 \mathrm{~g})$ by the size of the study area. The size of the study area varied because we always incorporated 10-12 focal groups (this included both breeding groups and bachelor groups) for study, and depending on population density, these groups had larger or smaller territories influencing the size of the study area. For each year, we 
determined the breeding season as the period when striped mice initiated breeding ( 6 weeks before pups emerged) to when breeding terminated ( 6 weeks before last observed pups; Brooks 1982; Schradin and Pillay 2005a). To determine breeding intensity (breeding ratio) in the study population, we divided the number of pups born each month by the number of adult females present on the field site, giving us a continuous measure. Date of birth was calculated for juveniles (body mass below $30 \mathrm{~g}$ ) using their body mass at first capture and a population specific growth curve (Schradin, Schneider \& Yuen 2009b). Adult sex ratio was determined from the same data as number of adult males divided by number of adult females. These estimates included individuals from non-focal groups at the periphery of the study area, which did not carry transmitters, and which were not directly observed, but only trapped for information about competing neighbouring groups. In contrast, for the frequency of bachelor males, i.e. the percentage of males following this tactic, only data from males living in focal groups were used, since information about the individual social tactics was less reliable from males in neighbouring groups which were not included in nest observations and radio-tracking.

\section{Ethical note}

We adhered to the ASAB/ABS Guidelines for the Use of Animals in Research (Bee et al., 2020). Mice were captured and handled using protocols approved by the Animal Ethics Screening Committee of the University of the Witwatersrand (AESC clearance number: 2007/40/01).

\section{Statistical Analysis}

Data were analysed using R Studio V3.6.2 (R Development Core Team, 2006), with tests being accepted as statistically significant when $P \leq 0.05$. We assessed the likelihood of scrotality in the five male tactics and evaluated the season (breeding versus non-breeding) that kin and non-kin bachelors were most likely to occur using Pearson's Chi Squared Tests.

Next, we evaluated competitive ability by comparing body mass and age in the five tactics by fitting generalised linear models (GLM) with the "glm" function from the MASS package (Veneables and Ripley, 2002) to the data. For comparison between the different tactics, we standardized data for each year, by using data from the onset of the breeding season in September, as done in previous studies on roaming males (Schradin et al. 2009a). Thus, we calculated the mean body mass of all morning measurements for the month of September for each male. Age was calculated for each male (where data were available) from birth dates, which were calculated using a population specific growth curve based on body mass that is reliable for individuals with a body mass of less than $30 \mathrm{~g}$ (Schradin, Schneider \& Yuen 2009b). Mean body mass was the response variable, age and tactic were fixed effects and male ID and year were introduced as random factors. Following significant effects of age and tactic on male body mass, pairwise comparisons using Wilcoxon rank sum tests, adjusted for multiple comparisons with the Benjamini-Hochberg method (Dunn, 1964) were used to determine where differences were.

We then examined the influence of population density, sex ratio and breeding ratio on the occurrence of kin and non-kin bachelor groups by fitting GLMs, specifying a Poisson distribution. Here, the number of bachelors that occurred was the response, population density, sex ratio, breeding ratio and their interactions were introduced as fixed effects, and year was introduced as a random factor. Analysis of variance tests (ANOVAs) were used to assess: 1 ) whether male body mass changed during their time (tenure) as bachelors and whether this change was influenced by the tactic prior to becoming a bachelor and 2) whether the subsequent tactic employed by bachelors was influenced by the body mass at the time of switching. Finally, the influence of kinship and tactic employed prior to becoming bachelors on tenure (total duration spent as bachelors) was evaluated by fitting a GLM. Tenure (time) was introduced as the response while kinship and prior tactic were fixed effects. For all GLMs, we conducted backward model reduction to select the minimum adequate model. 
We observed 108 bachelors in 40 bachelor groups comprising of 2-5 individuals (mean mean \pm SD: 2.65 \pm 1.004 ) from 2006 to 2019. We identified two main types of bachelor males: (a) 60 males in 22 kin bachelor groups ( $56 \%$ ), in which all females had disappeared leaving behind the male group members (offspring and breeding male of the group); and (b) 48 males in 18 non-kin bachelor groups (44\%), which were formed by males originating from different natal groups (Fig 1). Overall, $70 \%$ of all bachelors had previously been philopatric.

Scrotality and seasonality of bachelors.

Independent of tactic, adult males at the start of the breeding season were more likely to be scrotal than non-scrotal $\left(\chi 2=74.39_{d f=4} ; P<0.001\right.$, Pearson's Chi square test). Specifically, $99 \%$ of breeders ( $N$ $=164), 99 \%$ of roamers, $(N=68), 66 \%$ of philopatrics $(N=162), 80 \%$ of kin bachelors $(N=15)$ and 86 $\%(N=11)$ of non-kin bachelors were scrotal. Kin bachelors were more common during the nonbreeding season than the breeding season ( $\chi 2=16.09_{d f=1} ; P<0.001$, Pearson's Chi square test; Fig 2 ). In contrast, non-kin bachelors were more frequent in the breeding season than the non-breeding season ( $\chi 2=16.09_{d f=1} ; P<0.001$, Pearson's Chi square test; Fig 2).

Influence of population density and sex ratio on the occurrence of bachelors

252

Sex ratio was a significant predictor of the number of kin bachelors that occurred (GLM, $\chi 2=11.81_{d f=1}$; $P=0.016)$ whereas breeding opportunities, population density and the interaction between population density and sex ratio were not significant predictors (GLM, $P>0.05$ ). Thus, more kin bachelors occurred in a population with a male biased sex ratio (Fig 3a). Conversely, for non-kin bachelors, population density was a significant predictor (GLM, $\left.\chi 2=18.32_{d f=1} ; P=0.009\right)$ while sex ratio, breeding ratio, population density or the interaction effects were not significant (GLM, $P>0.05$ ). Thus, more non-kin bachelors occurred in conditions of low to intermediate population density, but none at very high population densities (Fig 3b).

\section{Comparison of body mass and age in five tactics at the start of the breeding season}

Male body mass at the start of the breeding season (September) was significantly related to both tactic $\left(\mathrm{GLM}, P<0.001 ; \chi 2=93.38_{d f=4}\right)$ and age $\left(\mathrm{GLM}, P<0.001 ; \chi 2=59.12_{d f=1}\right)$. Pairwise comparisons of body mass in the tactics revealed that philopatrics were the lightest, breeders the heaviest and roamers were of intermediate body mass (Table 1; Fig. 4a). Additionally, we found that non-kin bachelors were significantly heavier than kin bachelors. Kin bachelors were significantly lighter than breeders and roamers but did not differ from philopatric males. Non-kin bachelors were significantly heavier than philopatrics, lighter than breeders but did not differ from roaming males

Similar results were observed when age was analysed by tactic (Table 1; Fig 4b). Pairwise comparisons revealed that philopatrics were the youngest, breeders the oldest, while roamers were of intermediate age. Kin bachelors did not differ in age from philopatrics but were younger than males of all other tactics. Non-kin bachelors were older than philopatrics and kin bachelors but did not differ in age from both breeding and roaming males.

\section{Post bachelor tactics}


Males significantly gained weight during their tenure as bachelors (Anova; $F=3_{d f=2} ; P=0.0339$, Fig 5 ). Total change in body mass during the bachelor tactic was significantly influenced by the tactic which males had employed prior to becoming bachelors (Anova; $F=3_{d f=2} ; P=0.034$, Fig 5). Specifically, kin bachelors which had been philopatric prior to becoming bachelors increased body mass the most; while kin bachelors that had previously been breeders showed the smallest increase in body mass (Fig $5)$. Kin bachelors were more likely to die or disappear from the population than non-kin bachelors $(P=$ 0.004, Fisher's Exact Test). Bachelors that did not disappear (presumably due to death) went on to become roamers and breeders (Fig 6). Of the 24 surviving kin bachelors, 16 became breeders and 8 became roamers. Kin bachelors that became breeders were not heavier than roamers (Anova; $F=$ $0.749_{d f=1} ; P=0.391$; Fig 6). Similarly, of the 32 surviving non-kin bachelors, 16 went on to become roamers and 16 went on to become breeders, again with no significant difference in body mass (Anova; $\mathrm{F}=1.21_{\mathrm{df}=1} ; P=0.283 ;$ Fig 6$)$.

\section{Tenure of bachelor groups}

290

Overall, bachelor groups were maintained for a period of 1-5 months $(1.88 \pm 1.31)$ after which they disbanded (Fig 7). Tenure in bachelor groups was longest for kin philopatrics (GLM, $P<0.001,2.274 \pm$ 1.39), shortest in kin breeders (GLM, $P=0.0316,1.59 \pm 1.060$ ) and was not significantly influenced by other tactics (GLM, $P>0.05$ ), with males in non-kin bachelor groups having a similar tenure independent of their previous tactic (Fig. 6).

\section{DISCUSSION}

Males in several animal species exhibit variation in social and reproductive behaviour, which is often associated with differences in body condition. For example, young and old, small and large males regularly make use of alternative behavioural tactics in order to optimise their fitness (Gross 1996; Taborsky 2008). The occurrence of bachelor groups has recently been reported in the second largest land mammal (i.e. Asian elephants, Elephas maximus), where they represent a unique life history strategy for male elephants that are confronted by landscapes that have been altered by anthropogenic activities (Srinivasaiah et al. 2019). Here, we report for the first time, the existence of bachelor groups in a mammalian species that is much less than $1 \mathrm{~kg}$ in body mass and provide evidence that it represents an important life history stage for males of intermediate competitive ability.

Using data from 13 generations, we first showed that bachelor groups in the socially flexible African striped mouse arise as a consequence of two main mechanisms: 1) through the disappearance of all group females, leaving behind kin males in a bachelor group; and 2) when unrelated males form bachelor groups. We then showed that both kin and non-kin bachelors differ in seasonality of occurrence and in traits which are known indicators of competitive ability in striped mice, i.e. body mass, age, and scrotality (Schradin et al 2009a). Specifically, kin bachelor groups were more common during the non-breeding season and comprised of the large breeding male, which soon disappeared or moved into another group, and small philopatric males, which remained bachelors until the next breeding season. Non-kin bachelors were most common during the breeding season, nearly all of them were reproductively active, and their body mass was similar to that of solitary roamers. Finally, we found that non-kin bachelors had a short tenure and, after increasing their body mass, soon switched to the solitary roaming or the group-living breeder tactic. This indicates that non-kin bachelors might represent an alternative reproductive tactic that is similar to roaming males, but with the advantage of social thermoregulation by huddling in a nest at night, which is known to be an important mechanism for reducing energy expenditure in this species (Scantlebury et al, 2006).

In support of our expectations, two types of bachelor groups occurred, arising as a result of two different mechanisms. Firstly, we observed bachelor groups that formed as a result of environmental disrupters (Schradin 2013); where individuals were forced into the bachelor tactic (kin bachelor 
groups). Kin bachelor groups formed when all females of a group disappeared (presumably due to predation), leaving behind males. Secondly, we observed bachelor groups that formed due to social flexibility, where individuals can switch reversibly between tactics (Schradin et al., 2012). These were the non-kin bachelor groups, which consisted of unrelated males that originated from different groups. Importantly, we report that there was one instance where two related males left their natal group to form a bachelor group. More than half of bachelor groups (56\%) formed due to environmental disrupters (Schradin 2013), indicating that following the bachelor tactic often does not reflect individual male choices to form groups consisting of males only, but may be the result of stochastic extrinsic factors. Similar observations where stochastic extrinsic factors such as the death of important group members impose changes in tactics and social organisation (Schradin, 2018) have been reported in other species. For example, in pair-living Scandinavian wolves (Canis lupus lupus), the death of one of the pair members results in the other becoming temporarily solitary (Milleret et al. 2017). Similarly, in striped mice, the death of all but one adult female group member gives rise to female solitary breeders (Hill et al. 2015). Whether an alternative tactic is due to social flexibility or environmental disrupters can have significant influences on physiology (Hill et al. 2015b) and is also expected to influence fitness (Hill et al. 2015a).

At the start of the breeding season, both kin and non-kin bachelors were sexually mature and physiologically capable of breeding, as indicated by them being scrotal (Schradin et al. 2012). Since male striped mice cannot obtain a breeding position within their natal groups (Schradin et al. 2010), reproductive opportunities during the breeding season may encourage males to disperse. This may explain the significantly higher incidence of non-kin bachelor males in the breeding season than in the non-breeding season. Conversely, more kin bachelor males were observed during the non-breeding season than in the breeding season. This is likely due to the non-breeding season being much longer (nine months) than the breeding season (three months) which would make the death of all females in a group more likely, subsequently leading to a higher likelihood of the occurrence of kin bachelor groups. Similarly, a male-biased sex ratio was a significant predictor of number of kin bachelor groups that occurred. For non-kin bachelors, population density was a significant predictor, with more bachelors occurring in conditions of low to intermediate population density. This indicates that demographic factors may play an important role in constraining dispersal. A high population density may result in a lack of available territories (Emlen 1982a; Bergmuller et al. 2005), which hinders sexually mature individuals from choosing a dispersal tactic. Thus, reproductive competition during the breeding season, coupled with empty territories into which males can disperse (low population density) seem to favour dispersal leading to the formation of non-kin bachelor groups.

Body condition, reflected by an individual's age, body mass or size is an important factor in the determination of reproductive tactics in many species (Brockmann \& Taborsky 2008; Gross 1996), including striped mice (Schradin et al. 2009a). Interestingly, we found that non-kin bachelors were significantly heavier than kin bachelors and philopatrics, lighter than breeders but did not differ in body mass from roaming males. The finding that males which choose to disperse and become bachelors (non-kin bachelors) were heavier and older than males which were forced to become bachelors (kin bachelors) lies within our expectations since similar findings have been reported in female striped mice. Group-living females which became solitary by leaving a communal group differed in individual traits from those forced to do so by environmental disrupters by being heavier and having lower stress hormone levels (Hill et al. 2015). That individuals of the same observed behavioural tactic can differ in important traits such as body mass, age, and hormone levels in both male and female striped mice highlights the importance of distinguishing between environmental disrupters versus social flexibility as mechanisms leading to these tactics.

In male striped mice, the largest males are dominant breeders that defend territories wherein there are groups of two to four breeding females (Schradin et al 2009a). Thus, body mass is important for territory defence as well as for the maintenance of social and reproductive tactics (Schradin et al 2009a). Non-kin bachelors may thus be seen as males which are large enough to disperse, possibly due to the pressures of within-group reproductive competition (Schradin et al 2009b), but not competitive 
enough to obtain and defend a territory. This is common in many species, where forming bachelor groups is a tactic employed by less competitive males which may be excluded by territorial or resident males (Gartlan 1974; Owen-Smith 1975). These bachelor males form coalitions while they wait to secure a reproductive position in a group (e.g. patas monkey Erythrocebus patas; Gartlan 1974) or while they wait to gain a territory (e.g. white rhinoceros Ceratotherium simum; Owen-Smith 1975). In our study, tenure of non-kin bachelor males was short compared to kin-bachelors and most males switched soon to another tactic (some disappeared), indicating that this also represents an intermediate life history tactic.

A change in body condition is known to elicit a change in social or reproductive tactic in some species (John 1993; Hill et al., 2015). This is also true for male striped mice, which begin their life as philopatrics and can later switch to either the roaming or breeding tactic when their body mass increases (Schradin et al 2009b). Our results revealed that males significantly increased their body mass while in bachelor groups. Kin bachelors that had previously been philopatric gained the most body mass while in bachelor groups, and they also had the longest tenure. Kin bachelors that had previously been breeders were able to leave bachelor groups the fastest (had the shortest tenure), possibly because they were already large breeding males before the disappearance of all females, and thus did not need extended periods of time to gain body mass and become more competitive for a tactic change. Non-kin bachelors also gained significant body mass, although in a relatively shorter tenure as kin bachelors. Interestingly, while most kin bachelors disappeared from the population (61\%), most non-kin bachelors $(66 \%)$ managed to switch to the roaming or breeding tactic; tactics which are known to lead to reproductive success (Schradin et al. 2012). These results predict that non-kin bachelors should have significantly higher fitness than kin bachelors, and that the roaming tactic likely confers higher fitness than the bachelor tactic.

Alternative reproductive tactics (ARTs) occur where investment in reproduction can be exploited by competitors of the same sex (Taborsky 2008), such that high reproductive competition leads to the evolution of alternative tactics to obtain reproductive opportunities, especially for less competitive males (Alonzo 2008). Whether non-kin bachelor groups in striped mice represent a real ART remains unknown, since we do not know the reproductive consequences. Nevertheless, it is interesting to note that some males chose to form bachelor groups over the alternatives to either stay at home as philopatric males or to become solitary roamers. Remaining a philopatric male is associated with extremely low reproductive success, which is ten times higher in roamers (Schradin \& Lindholm 2011), which might explain why older and heavier males leave their natal groups and become roamers, even though this is associated with high energetic costs (Rimbach et al. 2019). One of the main reasons of group-living in striped mice is reduced costs of thermoregulation at night, since sleeping in huddling groups decreases these costs by up to $30 \%$ (Scantlebury et al. 2006). Thus, we posit that non-kin bachelor groups are best interpreted as an ART similar to the roaming tactic but with reduced energetic costs of solitary sleeping. Kin bachelors comprise of mostly previously philopatric males, have the longest tenure and gain the most body mass during their tenure as bachelors. Thus, we posit that these are very small males that might otherwise have remained philopatric until at least the next breeding season but were forced to be bachelors by environmental disrupters.

\section{Conclusions}

Here we report, for the first time, bachelor groups in the striped mouse, by far the smallest mammal for which this male tactic has been reported. Previous findings demonstrated that female striped mice which breed solitarily because they left their communal groups differ from those forced to do so by environmental disrupters (Hill et al. 2015a, b). The current study now shows that distinguishing the conditions under which striped mice males become bachelors also has important implications for individual traits. Our results indicate that individuals following the same behavioural tactic can differ in life history traits depending on the mechanism that gave rise to observed tactic, which should also be considered in other species with alternative tactics. 


\section{ACKNOWLEDGEMENTS}

This study was made possible with the support of the Succulent Karoo Research Station (registered South African NPO 122-134), Goegap Nature Reserve and several field assistants. We are thankful to numerous field assistants and students that helped in data collection over the years. This study was supported by the University of the Witwatersrand, the National Research Foundation [grant number 87769]; and the CNRS.

\section{DECLARATION OF INTERESTS}

The authors declare no conflicting interests.

\section{REFERENCES}

Alonzo, S. H. 2008. Conflict between the sexes and alternative reproductive tactics within a sex. In: Alternative Reproductive Tactics (Ed. by R. F. Oliveira, M. Taborsky \& H. J. Brockmann), pp. 435-450. Cambridge: Cambridge University Press.

Bee, M., Bernal, X., Calisi, R., Carere, C., Carter, T., Fuertbauer, L., Ha, J.C., Hubrecht, R., Jennings, D., Metcalfe, N., Ophir, A.G., Ratcliffe, J.M., Roth II, T., Smith, A., Sneddon, L. and Vitale, A. 2020. Guidelines for the treatment of animals in behavioural research and teaching. Animal Behaviour 159, $\mathrm{i}-\mathrm{xi}$

Brockmann, H. J. \& Taborsky, M. 2008. Alternative reproductive tactics and the evolution of alternative allocation phenotypes. In: Alternative Reproductive Tactics (Ed. by R. F. Oliveira, M. Taborsky \& H. J. Brockmann), pp. 25-51. Cambridge: Cambridge University Press.

Burton, M. \& Burton, R. 1969. The international wildlife encyclopedia. New York: Marshall Cavendish Corp.

Caro, T. \& Collins, A. 2010. Male Cheetah Social Organization and Territoriality. Ethology, 74, 52-64. Gartlan, J. S. 1974. Adaptive aspects of social structure in Erythrocebus patas. Proceedings from the Symposia of the 5th Congress of the International Primatological Society 17-161 Tokyo, Japan Science Press., 17-161.

Gross, M. R. 1996. Alternative reproductive strategies and tactics: diversity within the sexes. Trends in Ecology and Evolution, 11, 92-98.

Hill, D. L., Pillay, N. \& Schradin, C. 2015a. Alternative reproductive tactics in female striped mice: heavier females are more likely to breed solitarily than communally. Journal of Animal Ecology, 84, 1497-1508.

Hill, D. L., Pillay, N. \& Schradin, C. 2015b. Alternative reproductive tactics in female striped mice: Solitary breeders have lower corticosterone levels than communal breeders. Hormones and Behavior, 71, 1-9.

Jaeggi, A. V., Miles, M. I., Festa-Bianchet, M., Schradin, C. \& Hayes, L. D. 2020. Variable social organization is ubiquitous in Artiodactyla and probably evolved from pair-living ancestors. Proceedings of the Royal Society B: Biological Sciences, 287, 20200035.

Jarman, P. J. 1974. Social-organization of antelope in relation to their ecology. Behaviour, 48, 215-\&. Kappeler, P. M. 2019. A framework for studying social complexity. Behavioral Ecology and Sociobiology, 73, 13. Kappeler, P. M. \& Schaik, C. P. v. 2002. Evolution of primate social systems. Int J Primatol, 23, 707740.

Koenig, A. \& Borries, C. 2001. Socioecology of Hanuman langurs: The story of their success. Evolutionary Anthropology: Issues, News, and Reviews, 10, 122-137.

le Roux, A. \& Bergman, T. J. 2012. Indirect rival assessment in a social primate, Theropithecus gelada. Animal Behaviour, 83, 249-255.

Lott, D. F. 1991. Intraspecific Variation in the Social Systems of Wild Vertebrates. New York: Cambridge University Press. 
475 Sapolsky, R. 2011. Male African elephants (Loxodonta africana) queue when the stakes are high. Ethology Ecology \& Evolution, 23, 388-397.

Ohsawa, H. 2003. Long-term study of the social dynamics of patas monkeys (Erythrocebus patas): Group male supplanting and changes to the multi-male situation. Primates; journal of primatology, 44, 99-107.

Rimbach, R., Blanc, S., Zahariev, A., Pillay, N. \& Schradin, C. 2019. Daily energy expenditure of males following alternative reproductive tactics: Solitary roamers spend more energy than group-living males. Physiology \& Behavior, 199, 359-365.

Roberts, S. J. 2017. Bachelor Males. The International Encyclopedia of Primatology, pp.1-2.

Robisson, P., Aubin, T. \& Bremond, J. C. 1993. Individuality in the voice of the emperor penguin Aptenodytes forsteri: adaptation to a noisy environment. Ethology, 94, 279-290.

Scantlebury, M., Bennett, N. C., Speakman, J. R., Pillay, N. \& Schradin, C. 2006. Huddling in groups leads to daily energy savings in free-living African four-striped grass mice, Rhabdomys pumilio. Funct Ecol, 20, 166-173.

Schradin, C. 2013. Intraspecific variation in social organization by genetic variation, developmental plasticity, social flexibility or entirely extrinsic factors. Philosophical Transactions of the Royal Society B-Biological Sciences, 368, doi:10.1098/rstb.2012.0346.

Schradin, C. \& Lindholm, A. K. 2011. Relative fitness of alternative male reproductive tactics in a mammal varies between years. Journal of Animal Ecology, 80, 908-917.

Schradin, C., Lindholm, A. K., Johannesen, J., Schoepf, I., Yuen, C.-H., König, B. \& Pillay, N. 2012. Social flexibility and social evolution in mammals: a case study of the African striped mouse (Rhabdomys pumilio). Molecular Ecology, 21, 541-553.

Schradin, C. \& Pillay, N. 2004. The striped mouse (Rhabdomys pumilio) from the succulent karoo of South Africa: A territorial group living solitary forager with communal breeding and helpers at the nest. J Comp Psychol, 118, 37-47.

Schradin, C., Scantlebury, M., Pillay, N. \& König, B. 2009a. Testosterone levels in dominant sociable males are lower than in solitary roamers: physiological differences between three male reproductive tactics in a sociably flexible mammal. Am Nat, 173, 376-388.

Schradin, C., Schneider, C. \& Yuen, C. H. 2009b. Age at puberty in male African striped mice: the impact of food, population density and the presence of the father. Functional Ecology, 23, 10041013.

Shultz, S., Opie, C. \& Atkinson, Q. D. 2011. Stepwise evolution of stable sociality in primates. Nature, 479, 219-222.

Srinivasaiah, N., Kumar, V., Vaidyanathan, S., Sukumar, R. \& Sinha, A. 2019. All-Male Groups in Asian Elephants: A Novel, Adaptive Social Strategy in Increasingly Anthropogenic Landscapes of Southern India. Scientific Reports, 9, 8678.

Taborsky, M. 2008. The evolution of alternative reproductuve tactics: concepts and questions. In: Alternative Reproductive Tactics (Ed. by R. F. Oliveira, M. Taborsky \& H. J. Brockmann), pp. 1-21. Cambridge: Cambridge University Press.

Waterman, J. 1997. Why do male Cape ground squirrels live in groups? Animal Behaviour - ANIM BEHAV, 53, 809-817.

Westcott, D. 1994. Leks of Leks: A Role for Hotspots in Lek Evolution? Proceedings of the Royal Society B: Biological Sciences, 258, 281-286.

Wirtz, P. 1982. Territory Holders, Satellite Males and Bachelor Males in a High Density Population of Waterbuck (Kobus ellipsiprymnus) and their Associations with Conspecifics1). Zeitschrift für Tierpsychologie, 58, 277-300.

521 
Table 1: Results of post-hoc pairwise comparisons of body mass (g) and age (months) across different male tactic in Rhabdomys pumilio using the Wilcoxon rank sum test for multiple comparisons, with the Benjamini Hochberg p-adjustment method. Significant differences between tactics appear in bold.

$$
\begin{array}{llll}
\text { Philopatric Breeder } & \text { Roamer } & \begin{array}{l}
\text { Kin } \\
\text { bachelor }
\end{array} & \begin{array}{l}
\text { Non-kin } \\
\text { bachelor }
\end{array}
\end{array}
$$

\begin{tabular}{lllllll}
\hline Philopatric & Mass & - & $\boldsymbol{P}<\mathbf{0 . 0 5}$ & $\boldsymbol{P}<\mathbf{0 . 0 5}$ & $\boldsymbol{P}>0.05$ & $\boldsymbol{P}<\mathbf{0 . 0 5}$ \\
& Age & - & $\boldsymbol{P}<\mathbf{0 . 0 5}$ & $\boldsymbol{P}<\mathbf{0 . 0 5}$ & $\boldsymbol{P}>0.05$ & $\boldsymbol{P}<\mathbf{0 . 0 5}$ \\
\hline Breeder & Mass & $\boldsymbol{P}<\mathbf{0 . 0 5}$ & - & $\boldsymbol{P}<\mathbf{0 . 0 5}$ & $\boldsymbol{P}<\mathbf{0 . 0 5}$ & $\boldsymbol{P}<\mathbf{0 . 0 5}$ \\
& Age & $\boldsymbol{P}<\mathbf{0 . 0 5}$ & - & $\boldsymbol{P}<\mathbf{0 . 0 5}$ & $\boldsymbol{P}<\mathbf{0 . 0 5}$ & $\boldsymbol{P}>0.05$ \\
\hline Roamer & Mass & $\boldsymbol{P}<\mathbf{0 . 0 5}$ & $\boldsymbol{P}<\mathbf{0 . 0 5}$ & - & $\boldsymbol{P}<\mathbf{0 . 0 5}$ & $\boldsymbol{P}>0.05$ \\
& Age & $\boldsymbol{P}<\mathbf{0 . 0 5}$ & $\boldsymbol{P}<\mathbf{0 . 0 5}$ & - & $\boldsymbol{P}>0.05$ & $\boldsymbol{P}>0.05$ \\
\hline Kin bachelor & Mass & $\boldsymbol{P}>0.05$ & $\boldsymbol{P}<\mathbf{0 . 0 5}$ & $\boldsymbol{P}<\mathbf{0 . 0 5}$ & - & $\boldsymbol{P}<\mathbf{0 . 0 5}$ \\
& Age & $\boldsymbol{P}>0.05$ & $\boldsymbol{P}<\mathbf{0 . 0 5}$ & $\boldsymbol{P}>0.05$ & - & $\boldsymbol{P}>0.05$ \\
\hline Non-kin bachelor & Mass & $\boldsymbol{P}<\mathbf{0 . 0 5}$ & $\boldsymbol{P}<\mathbf{0 . 0 5}$ & $\boldsymbol{P}>0.05$ & $\boldsymbol{P}<\mathbf{0 . 0 5}$ & -
\end{tabular}



Figure 1: The number of bachelor males in each origin category. Bachelor groups formed when all females of a breeding group disappeared, leaving behind only males (kin bachelors) or when unrelated males that had dispersed from their natal groups formed a bachelor group (non-kin bachelors). The pre-tactics of bachelor males are indicated. Bar width indicates group sample size. 


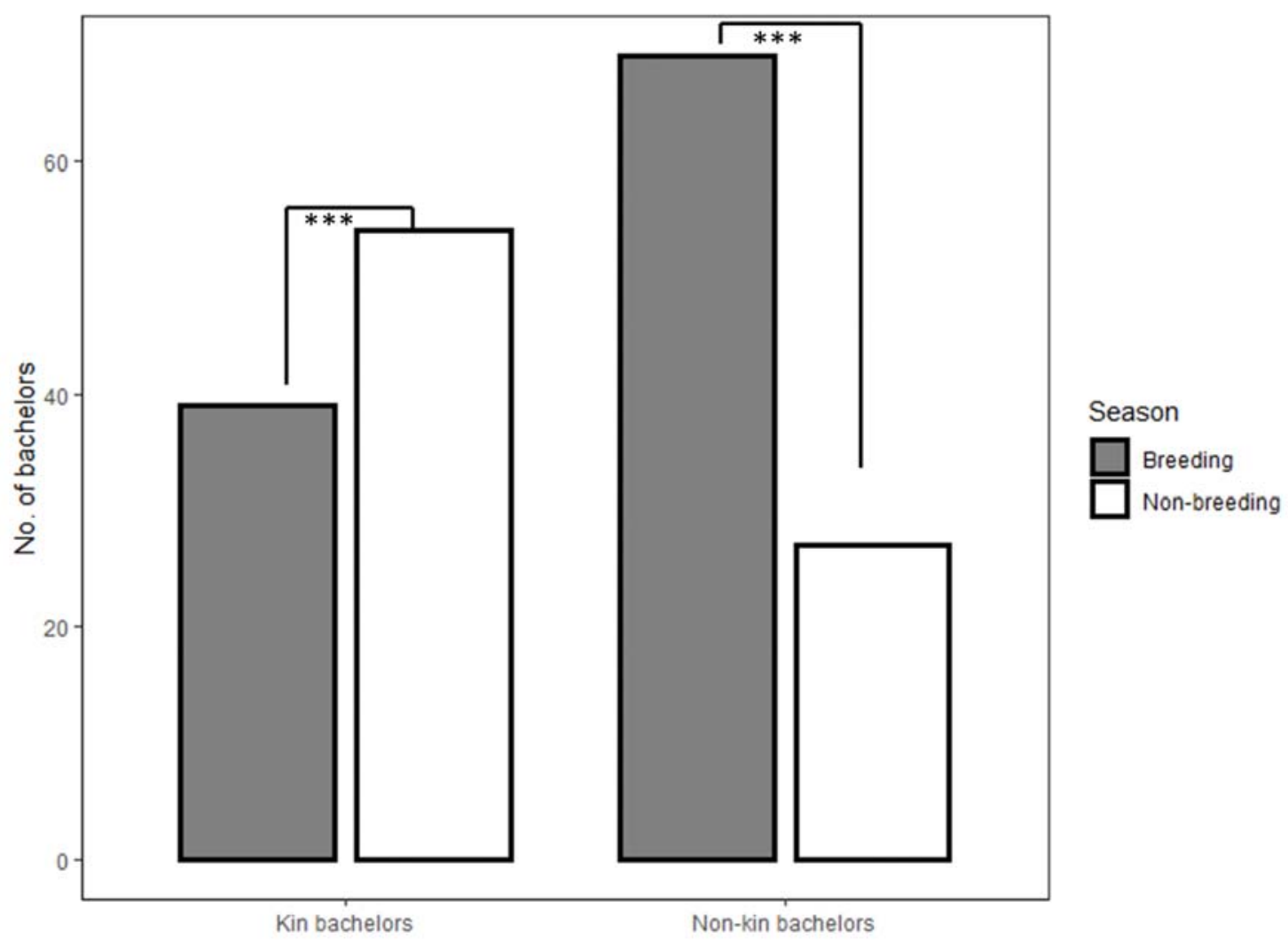

Figure 2: The number of kin and non-kin bachelors that occurred during the breeding season and the non-breeding season. ${ }^{* * *}$ denote statistically significant differences between the seasons.
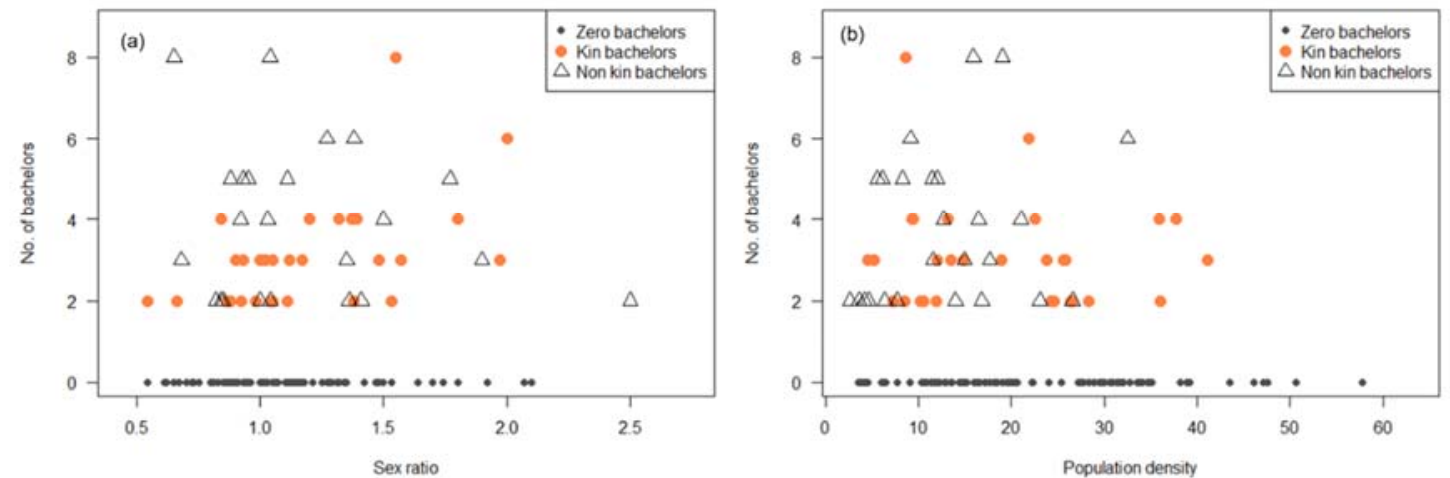

Figure 3: Body mass (a) and age (b) of philopatric, breeding, roaming, kin bachelors and non-kin bachelors at the start of the breeding season (September). Horizontal lines inside the box show the median, whiskers show minimum and maximum values. Black circles show the outliers. Different letters above box plots depict statistically significant differences following the Wilcoxon pairwise comparisons adjusted for multiple comparison with the Benjamini Hochberg method. 



Figure 4: (a) Number of kin and non kin bachelors that occurred at different sex ratios (number of adult males/number of adult females) and (b) Number of kin and non-kin bachelors that occurred at different population densities (total number of adult mice per hectare).



Figure 5: Total change in body mass ( $\mathrm{g} /$ full period) in kin and non-kin bachelors during time spent highest increase in body mass amongst all bachelors $(P<0.001)$. 


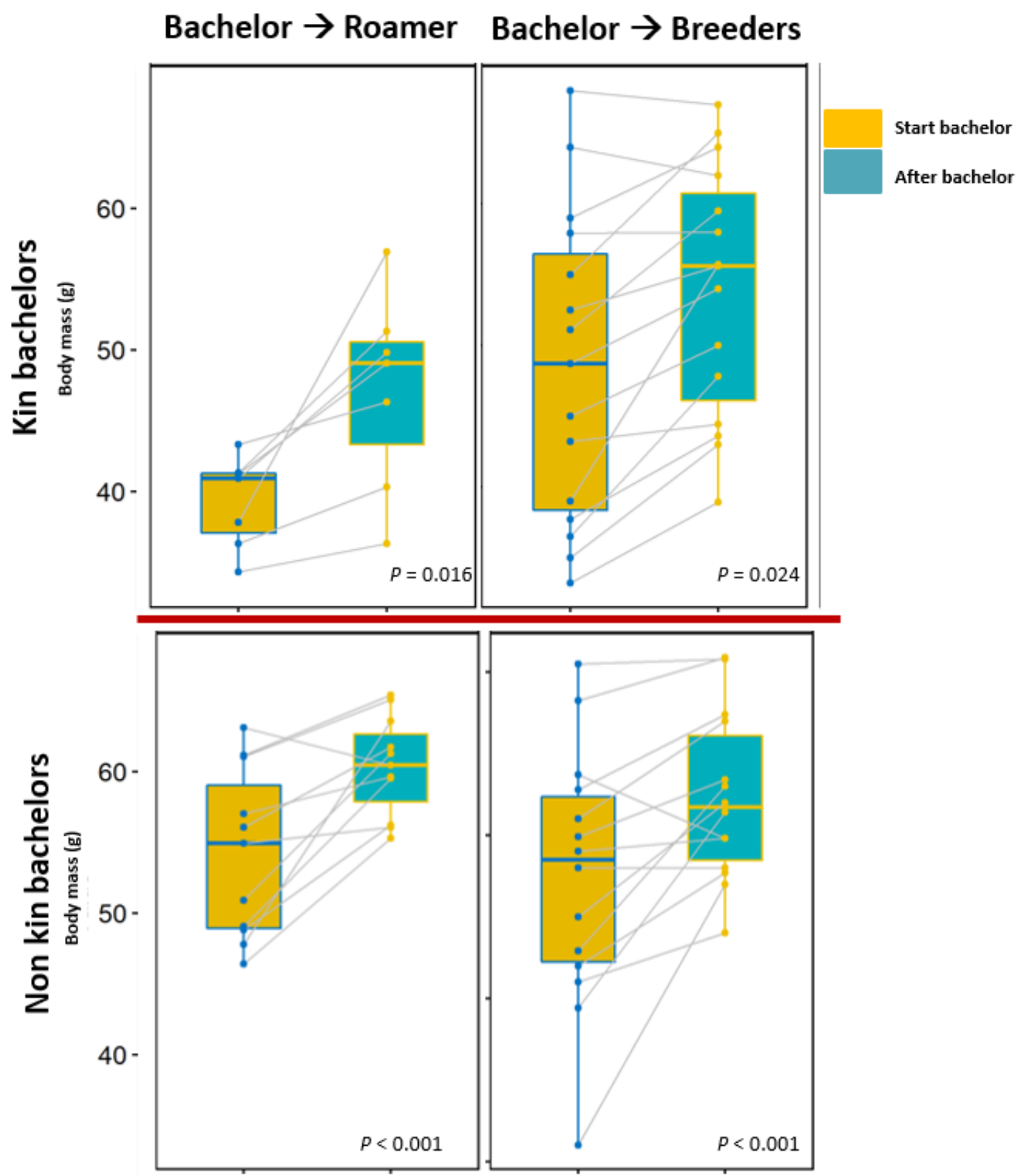

Figure 6: Change in body mass during tenure as bachelors. The boxplots compare the mean body mass of males when they switched to the bachelor tactic (yellow boxes) and when they switched from bachelor (blue boxes) to breeding or roaming tactic. Horizontal lines inside the box show the median, whiskers show minimum and maximum values. Grey lines depict changes in body mass before and after becoming bachelors for individual males. P-values are indicated inside frames for each comparison following Wilcoxon rank sum tests. 


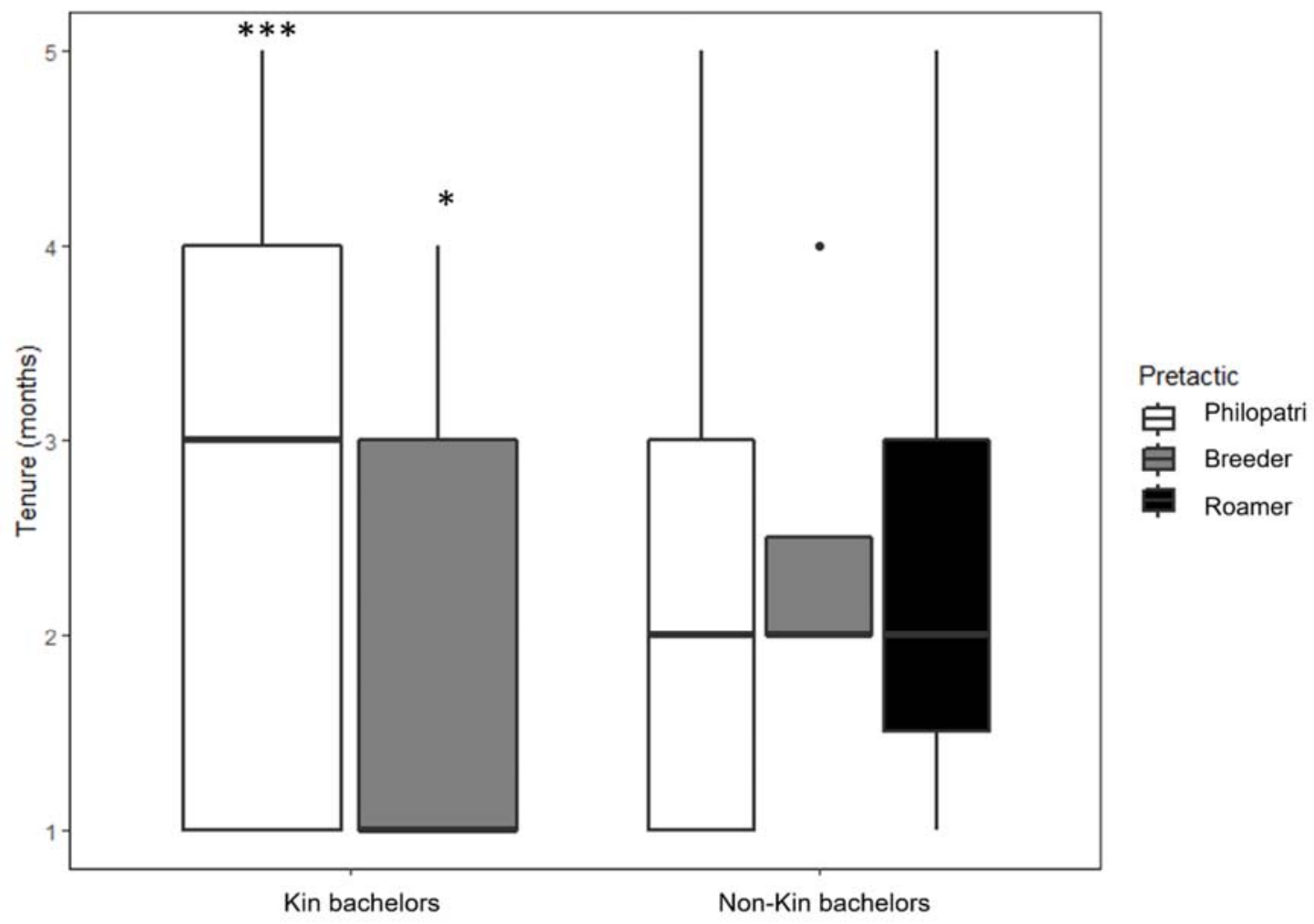

Figure 7: The tenure (in months) that males remained in bachelor groups before switching to another 567 tactic (or disappearing from the study site). Horizontal lines inside the box show the median, whiskers 568 show minimum and maximum values. Asterisks $(*)$ show the variables that significantly influenced the 569 duration spent as bachelors (GLM; $\left.{ }^{*} \mathrm{p}<0.05 ; * * * p<0.001\right)$. 